\title{
Capabilities of ARIANNA: Neutrino Pointing Resolution and Implications for Future Ultra-high Energy Neutrino Astronomy
}

\author{
Steven W. Barwick ${ }^{a, *}$ and Geoffrey Gaswint ${ }^{a}$ on behalf of the ARIANNA \\ Collaboration \\ (a complete list of authors can be found at the end of the proceedings) \\ ${ }^{a}$ University of California, Department of Physics and Astronomy, \\ Irvine, CA, 92617, USA \\ E-mail: sbarwick@uci.edu, ggaswint@uci.edu
}

\begin{abstract}
A radio-frequency polarization measurement by the ARIANNA surface station was performed using a residual hole from the South Pole Ice Core (SPICEcore) Project. Radio pulses were emitted from a transmitter located down to $1.7 \mathrm{~km}$ below the snow surface. After deconvolving the raw signals for the detector response and attenuation from propagation through the ice, the signal pulses show no significant distortion and agree with a reference measurement of the emitter made in an anechoic chamber. The direction to transmitted radio pulse was measured with an angular resolution of 0.37 degree [statistical error]. For polarization, the statistical error of the polarization vector is depth dependent and below 1 degree. In addition, a slowly-varying systematic variation is observed as a function of depth with an rms error of 2.7 degrees. Neither the direction or polarization measurement show a significant offset as a function of depth relative to expectation. We also report on the results of a simulation study of the ARIANNA neutrino direction and energy resolution. The software tool NuRadioMC was used to reconstruct the polarization and viewing angle to determine the neutrino direction. Multiple models of Askaryan radiation and detector sites along with a range of neutrino energies were evaluated. The neutrino space angle resolution was determined to be below 3 degrees, which is comparable to the systematic polarization uncertainty. Therefore it is expected that the polarization resolution, which is the dominant contribution to the neutrino space angle resolution, will be improved in future studies by determining and eliminating systematic effects.
\end{abstract}

$37^{\text {th }}$ International Cosmic Ray Conference (ICRC 2021)

July 12 th - 23rd, 2021

Online - Berlin, Germany

\footnotetext{
${ }^{*}$ Presenter
} 


\section{Introduction}

During the last decade, several ultra-high energy (UHE) neutrino detector designs (for the purposes of this paper, a UHE neutrino is defined as neutrino energy, $E_{v}$, in excess of $10^{17} \mathrm{eV}$ ) based on the detection of short duration radio pulses have been tested in the pilot phase. Over the same time, the observation of gravitational waves and high energy neutrinos have provided the astrophysics community with the potential to observe sources emitting a variety of messengers, each providing its own insights on the physics mechanisms. To contribute to the growing effort in multi-messenger astronomy, radio-based neutrino detectors must determine the direction and energy of the neutrino event with as much precision as possible. Reducing the angular uncertainty will simplify the search for concurrent observations from high energy gamma ray telescopes, lower energy neutrino telescopes, gravitational wave detectors and potentially UHE cosmic ray detectors such as the Pierre Auger Observatory and Telescope Array. The time scale for neutrino emission is highly uncertain. Explosive sources may emit neutrinos on $O(1)$ second or shorter, and transient or flaring time scales may last for years. The best studied source observed by IceCube, TSX0506+056, emitted neutrinos within a flaring period that extended for months. A broad search over unknown timescales places a strong emphasis on developing technologies and analysis techniques that reduce the space angle resolution for all candidate events.

There is a second benefit from the measurement of angular direction because, as explained in section 2, it depends on the measurement of the polarization direction of the electric field. Polarization, combined with angular direction of the propagating pulse, and distance to the vertex provide a self-consistent measurement of neutrino properties that are unlikely to be fulfilled by many background processes. As an example, the polarization direction of radio pulses produced by cosmic ray interactions in the atmosphere is predominantly horizontal, which helps to distinguish these events from neutrino signatures. Backgrounds may be identified from the computed angular direction. At energies relevant to ARIANNA, events that pass through the earth are incompatible with standard model neutrino properties and likely background. Since the goal of the next generation of radio-based neutrino telescopes is to improve the sensitivity for neutrino detection by four orders of magnitude (relative to the sensitivities achieved by pilot arrays), the identification and removal of exceedingly rare background events has high priority. The ingredients required to measure the neutrino direction with good precision provide additional handles to help identify unforeseen background processes.

A pilot array of the ARIANNA high energy neutrino detector is located at Moore's Bay, within the Ross Ice Shelf in Antarctica [1]. Its near surface design streamlines the measurement of polarization, vertex distance, shower energy, and neutrino direction with only five antenna channels. Two station configurations have been studied for an assessment of neutrino direction resolution [2]: (1) an 8 channel design consisting of 4 downward facing LPDA buried to a depth of $1 \mathrm{~m}$ and four vertically oriented dipoles at a depth of $1 \mathrm{~m}$, and (2) an 8 channel design consisting of 4 downward facing LPDA, and 1 dipole at a depth of $15 \mathrm{~m}$. Design (1) was installed at the South Pole at a distance of $650 \mathrm{~m}$ from a previously drilled ice core (SPice), and (2) was installed at Moore's Bay (station 51). Configuration (2) was studied for both the Moore's Bay site and South Pole site .

It is desirable to compare the flux of neutrinos measured by independent techniques. Over much of the energy regime targeted by ARIANNA, another technique has been developed that 
installs antennas between $100 \mathrm{~m}$ and $200 \mathrm{~m}$ beneath the snow surface. The deep ice technique provides more volume per station, but more limited options for antenna receivers (since constrained to fit in a relatively narrow hole) and highest cost per hole. The deep ice relies on a volumetric reconstruction that differs from the ARIANNA technique that relies on area based reconstruction using directional LPDA antennas.

At lower energies, it is conceivable that radio techniques will overlap with optical neutrino detectors, especially if the next generation of larger optical Cherenkov detectors are constructed. Not only would a detailed comparison between the two detector architectures be immensely valuable in boosting confidence in the radio results, but radio could complement optical by providing an independent measurement of the angular direction, perhaps with comparable angular resolution for some classes of events, for sources in the southern sky.

It is notoriously difficult to predict the antenna response over all angles and frequencies when it is embedded in a non-uniform medium. The upper $100 \mathrm{~m}$ of Antarctic ice varies in density with the largest gradients near the surface. In addition, nearby discontinuities from the air-snow boundary will create additional challenges to modeling the antenna response. The ARIANNA station was designed to minimize many systematic issues associated with antenna responses by installing the receivers in a symmetric geometric layout. Many systematic errors associated with antenna responses are eliminated in the measurement of time delays between parallel antennas using a cross-correlation technique that requires that both waveforms show similar shapes. The antenna layout has an important impact on the resolution of measurable quantities such as angular direction of the radio pulse, the vertex resolution or neutrino angular resolution. The same argument holds for the rest of the front end signal chain such as amplifiers, cable effects, and readout electronics. The 4 fold symmetry provides two parallel measurements for two orthogonal polarization directions, minimizing the effects of systematic uncertainties in the response functions. Though the angular direction of the radio pulse can be triangulated from just three antennas, the antenna effects in mixed media near a discrete boundary must be understood with excellent precision.

An orthogonal geometry of LPDA antennas allows for the measurement of multiple polarization components and provides the ability to reconstruct the electric field. A vertically orientated dipole antenna, located at a depth of $15 \mathrm{~m}$, provides an important measurement of a time-separated direct and surface reflected signal within the same time record. Time delays between the direct and reflected signal are used to determine the distance to the source of the event. The timing resolution between direct and reflected signals was measured to be as good as 5 picoseconds for high signalto-noise ratios [3] because the effects of the antenna model, channel to channel time delay offsets, and amplifier response is again completely removed when measured by a common antenna.

\section{Directional and polarization reconstruction using in-situ pulser}

As discussed in [4], the angular direction of the neutrino is computed from the following information: (1) the polarization direction of the electric field, (2) the arrival direction of the radio pulse, (3) the location of the interaction vertex, (4) the angle of emission of the radio signal relative to the shower axis, which is within 20 degrees of the Cherenkov angle, and (4) refractive properties of the ice medium. An accurate ice model is required to propagate the arrival direction and polarization vector to the interaction vertex. The accuracy of these measured quantities was studied 

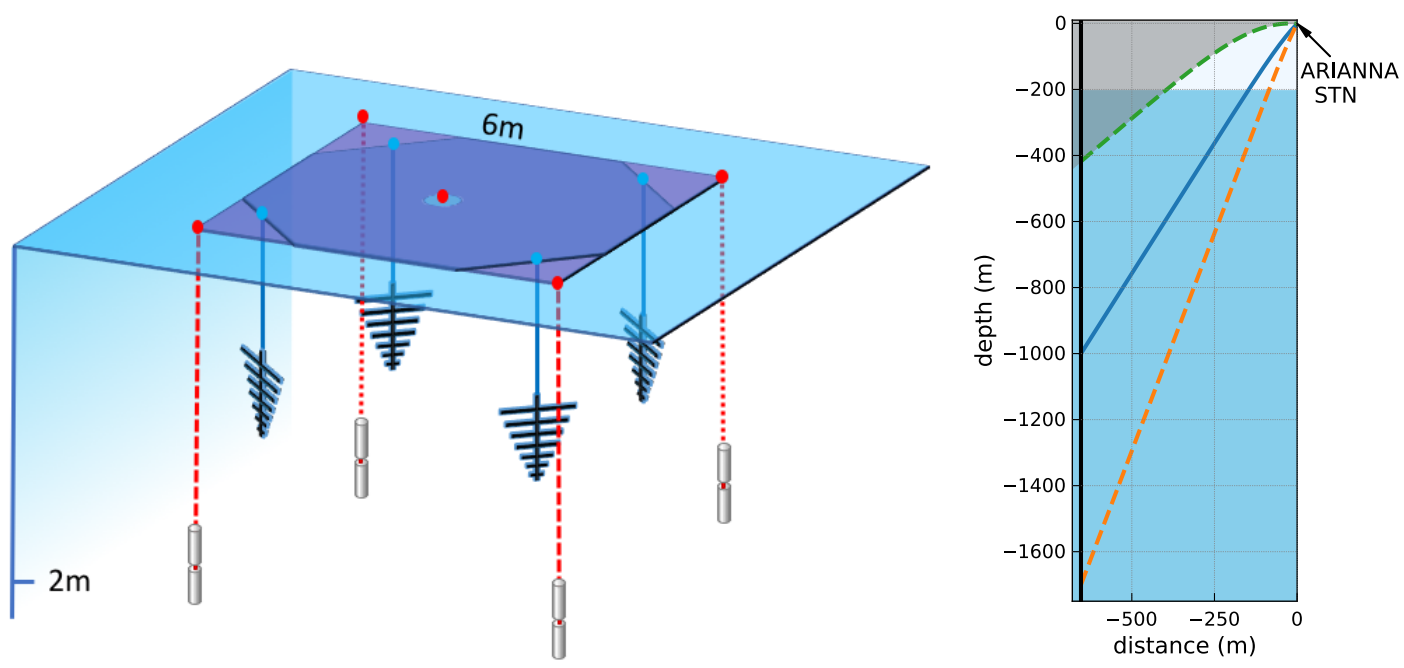

Figure 1: (left) Schematic illustration of ARIANNA Station 51 at South Pole. (right) Horizontal view of SPice hole geometry. Green dash demarcates the shadow zone. Blue and orange dash show two arbitrary paths.

in ARIANNA station 51 using in-situ radio pulses collected during the last week of December, 2018. Figure 1 shows a sketch of ARIANNA station 51 at the South Pole and the geometry between the SPice hole and station 51.

The signal transmitter (IDL-1 pulser) was connected to a bicone antenna which was lowered to a depth of $1.7 \mathrm{~km}$ inside the SPICE hole and was vertically-oriented (to match the form-factor of the SPICE hole) [5]. The IDL-1 pulser broadcasts short duration radio frequency pulses through the bicone antenna with a repetition rate of $1 \mathrm{~Hz}$, which is then detected by the ARIANNA station. Several thousand pulser events were directly transferred over the Iridium satellite network for offline analysis of the angular and polarization reconstruction capabilities of ARIANNA. The characteristics of the signal transmitter were tested in an anechoic chamber and combined with simulations of the known ice effects on signal propagation from transmitter to receiver in the SPICE run.

Station 51 is located roughly $1 \mathrm{~km}$ from South Pole Station and $0.65 \mathrm{~km}$ from the SPICE hole. Initially, the geometry of the bore hole was unavailable to us, so the first angular reconstruction results were first presented assuming no tilt in the SPICE hole [6]. Radio pulses were emitted from a transmitter in the SPice hole that was lowered from the surface to a depth $1.7 \mathrm{~km}$, the maximum depth of the borehole. After deconvolving the raw signals for the detector response and attenuation from propagation through the ice, the signal pulses show no significant distortion and agree with a reference measurement of the emitter made in an anechoic chamber. The direction to transmitted radio pulse was measured with an angular resolution of 0.37 degree [statistical error].

Recently, the SPiceCore Collaboration has provided the geometry of the hole as a function of depth. Fig. 2 shows the differences between measured and expected arrival directions, where the hole geometry was included (for details, see [2]). Compared to the earlier study, including the tilt profile slightly improves the angular reconstruction results. Uncertainties in the SPICE hole geometry translates to a systematic uncertainty in the relative position of the emitter with respect to 

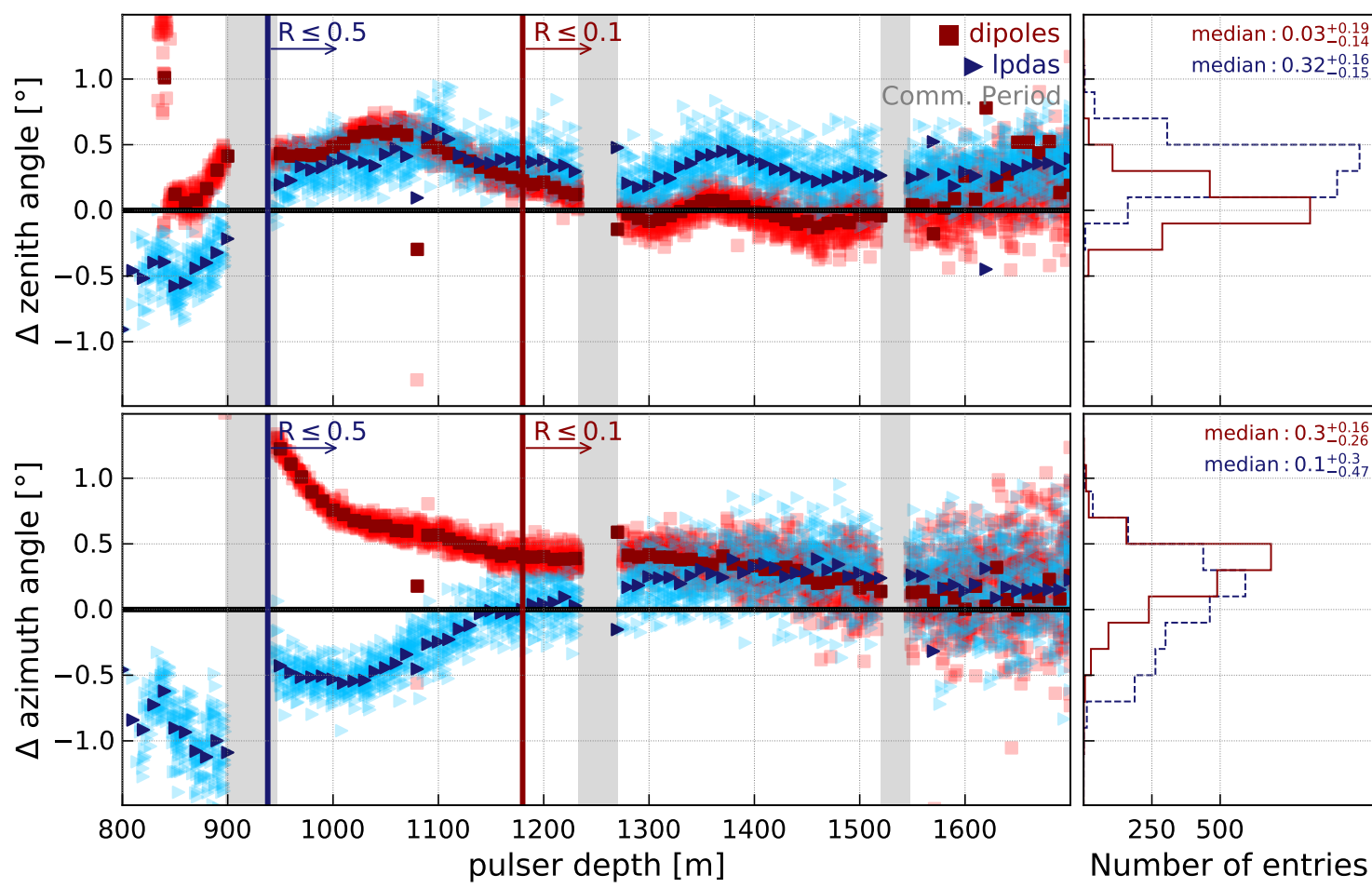

Figure 2: Difference between measured and expected arrival directions (top plots:zenith angle, bottom plots: azimuth angle). Left plots show the depth dependence; histogram projections are shown on the right. The expected direction includes the known geometry of the hole. Light blue triangles show the residuals using the four LPDAs along with a $10 \mathrm{~m}$ average shown in a darker blue color. Red squares show the residuals using the four dipoles along with a $10 \mathrm{~m}$ average shown in a darker red color. The red vertical line corresponds to a reflection coefficient from the snow surface of 0.1 , while the blue vertical line corresponds to a reflection coefficient of 0.5 . The gray shaded area indicates the periods where station 51 was in communication mode and thus not taking data. The projected histograms (LPDAs:blue dash, dipoles:solid red) present the residuals on an event-by-event basis (i.e. without the averaging).

the detector station, which in turn results in a systematic uncertainty in the predicted signal arrival direction. The depth dependence has flattened for depths greater than $1 \mathrm{~km}$, leading to improved zenith and azimuth resolutions by roughly $0.05^{\circ}$ for each antenna type. This demonstrates that the angular reconstruction by an ARIANNA station is sensitive to typical variation in the geometry of cored boreholes.

The linear polarization of the arriving radio pulse was determined as a function of depth and compared to expectation based on laboratory measurements of the transmitting pulser (Fig. 3, right). There is a slowly varying systematic error as a function of depth of 2.7 degrees. Statistical error in the polarization measurement is smaller than 1 degree, but the excellent precision is partly a consequence of the large signal to noise. Neither the direction nor polarization measurement show a significant offset as a function of depth relative to expectation. The right panel of Fig.3 shows the depth dependence of both polarization components. The relatively flat response of the theta component indicates good understanding of ice effects during propagation. Only the subdominant phi component shows a variation with depth which causes the variation in the polarization angle measurement. Laboratory calibration studies of the SPice transmitter show strong differences 

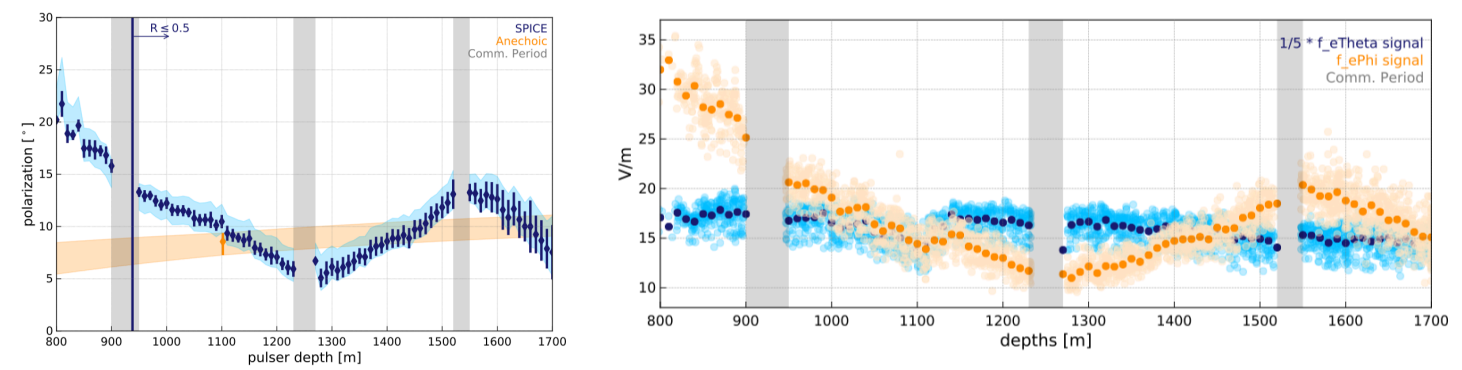

Figure 3: (left) Polarization angle as a function of depth (data). Yellow band shows expected polarization of transmitter (right) Polarization components as a function of depth. The theta component is scaled down by factor 5 . For additional details on these calculations, see Ref.[2]

between the theta and phi component in the frequency and time dependence of the electric field. This will not be the case for neutrino emission. It is possible that small errors in the angular emission emission model within a cylindrical borehole are responsible for the depth dependence in the phi polarization. Future work will focus on determining the cause of the systematic error.

\section{Monte Carlo study direction reconstruction of ARIANNA detector}

In-situ measurements described in the previous section and cosmic ray "test beams" [7] were used to validate many of the elements required to reconstruct the direction of the neutrino, including the signal arrival direction, the description of the ice properties to correct for the bending in the near surface ice, and signal polarization. However, in-situ measurements involved radio pulses with relatively large signal to noise ratio (SNR). In this section, we present the results of a simulation study using NuRadioMC [8] that evaluates the space angle resolution of the neutrino (where space angle is the angular difference between the reconstructed angular direction and the true angular direction of the neutrino) for a realistic event distribution generated by high energy neutrinos. The detector configuration is shown in left panel of Fig.4 which has been proposed for the next phase of ARIANNA [9] and suggested for the surface component of the radio neutrino array of IceCubeGen2[10]. Station 52 [7] of the ARIANNA pilot array, which has similar geometry, used cosmic rays as a "test beam" to study polarization resolution.

The simulation generated a random distribution of hadronic showers from neutrino interactions at specific energies in the South Pole ice. The detector triggers on the observed amplitude within a restricted frequency band [11] of $80-150 \mathrm{MHz}$ at threshold of $3.9 V_{r m s}$ in the downward LPDA, with a simple 2 of 4 majority logic. The reconstruction begins with the waveforms observed by the station receivers, and searches for a minimum in the global fit of angular parameters using the forward-folding method (see Fig. 5 left) [12]. A realistic distribution of the location of interaction vertices, due to reconstruction uncertainties [3], was included though this had small impact on the space angle resolution [2]. Electromagnetic showers initiated by $v_{e}$-CC interactions are in general more challenging to reconstruct, due to the LPM effect distorting the shower development, but a recent study using deep-learning techniques showed that also this event topology can be reconstructed with only a slight deterioration in angular resolution compared to hadronic showers [13]. 

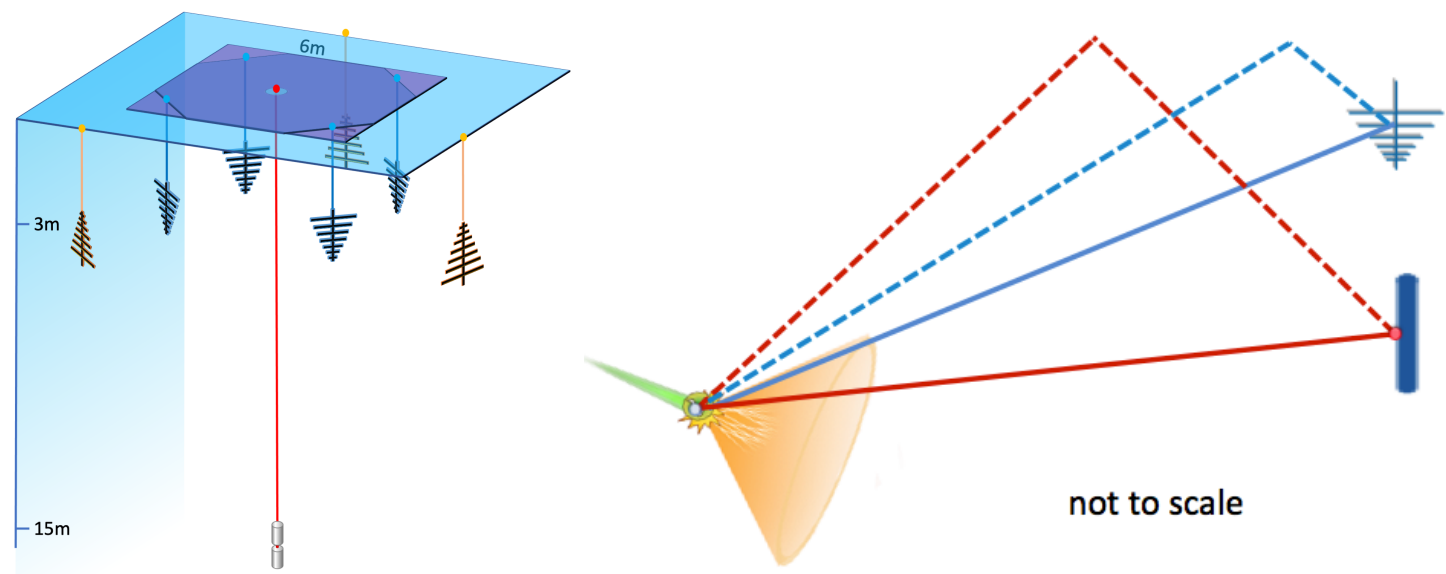

Figure 4: (left) Schematic of station geometry used in the simulation of space angle reconstruction. (right) Schematic of ray paths for one of the four downward facing LPDA (solid blue is direct path, blue dashed reflects off the snow-air surface) and the central dipole (solid red=direct, dashed red=reflected)
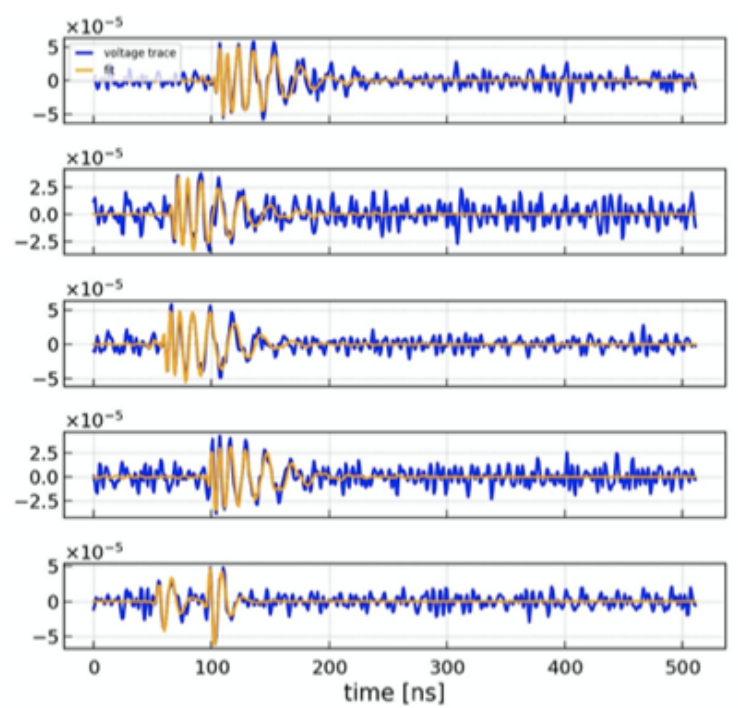

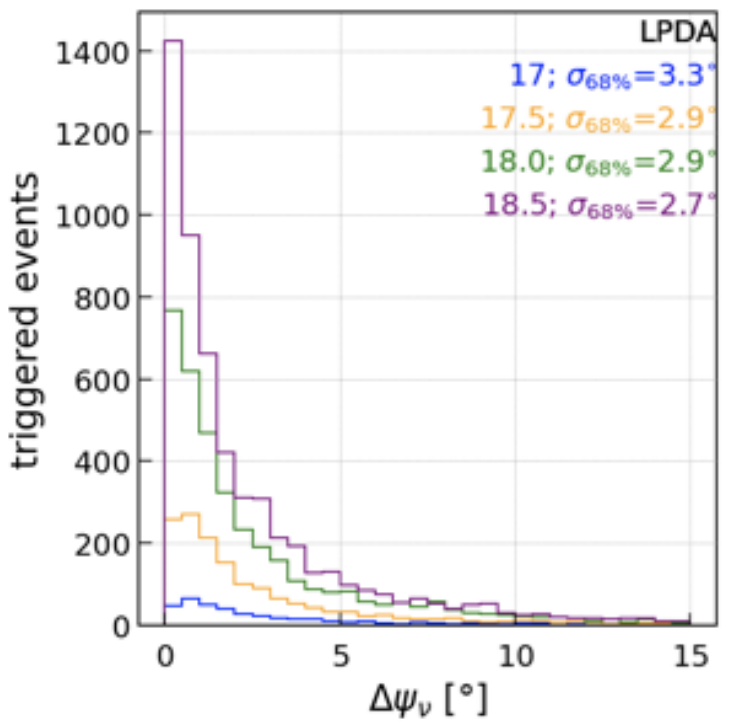

Figure 5: (left) Example event showing the amplitude variation in the time dependent signals in the LPDA (top 4 panels) and dipole (bottom panel). The double pulse structure in the dipole shows the time difference between the direct and reflected pulses. The yellow trace shows the outcome of global fit. To help visualize the event, the selected SNR was unusually high. (right) Distribution of space angle difference between reconstructed angular direction and true angular direction, $\Delta \Psi_{v}$ for several neutrino energies. The angle that contains $68 \%$ of the events is shown in legend.

The right panel of Fig. 5 shows the space angle resolution, which is given by the $68 \%$ containment parameter, $\sigma_{68 \%}$ for neutrino energies spanning from $10^{17} \mathrm{eV}$ to $10^{18.5} \mathrm{eV}$. The distributions show all triggered events without additional quality cuts. It shows that the space angle resolution of the surface station is approximately 3 degrees, with a small dependence due to energy. The limitation is primarily due to noise fluctuations in events with very small signals in the dipole or one of the pairs of LPDA or both. The angular resolution is improved by imposing an additional quality 
cut on the minimum amplitude in all four LPDA, but this also reduces the fraction of neutrino events in the analysis sample. The angular resolution can be improved by replacing the single dipole with a phased array of 4 or more dipoles [2] because of the improved SNR relative to a single dipole. Another improvement is expected when the back lobe response of the downward facing LPDA is included in the reconstruction. One final note. The studies of [2] show that the angular resolution of the viewing angle, which is the actual angle of emission that reaches the ARIANNA station relative to the angle of the Cherenkov cone, can be determined to an accuracy of 1 degree. The relatively small error can be attributed to the broad frequency response of the LPDA because the frequency spectrum depends on the viewing angle as well as the observation of the amplitude differences in the direct and reflected signal pulse observed in the dipole channel.

\section{Conclusions}

Reducing the error in the angular direction broadens the reach of multi-messenger searches for time variable sources. In addition, polarization is a valuable tool to identify rare, and perhaps unforeseen, background events that may arise in future detectors that expect to increase the sensitivity by factors of $10^{2}-10^{4}$ relative to current radio-based limits. Measurements of the signal arrival direction from in-situ pulser signals showed agreement with expectation to within $0.37^{\circ}$ after correcting for propagation effects and hole geometry, indicating good understanding of antenna response of the buried LPDA, index of refraction as a function of depth, and attenuation. It was expected that the ARIANNA design, which employs the same antenna type (LPDA) in orthogonal directions, would reduce the systematic errors in a polarization measurement. The same in-situ studies at the South Pole yielded $1^{\circ}$ resolution (stat) in the polarization direction, an encouraging result. The combination of these two studies, and an earlier study of the DNR method [3], validates critical components of the simulation tools. End-to-end simulation studies of the directional reconstruction capabilities of an ARIANNA-style surface detector gives a space angle (68\% containment) of 2.9 degrees for all triggered neutrino events (so no additional quality cuts were imposed) with $E_{v}=10^{18} \mathrm{eV}$. The space angle resolution varied weakly with energy.

\section{References}

[1] ARIANNA Collaboration, A. Anker et al. JCAP 03 (2020) 053.

[2] G. Gaswint, University of California, Irvine. PhD thesis, 2021.

[3] ARIANNA Collaboration, A. Anker et al. JCAP 11 (2019) 030.

[4] C. Glaser for the ARIANNA collaboration PoS ICRC2019 (2020) 899.

[5] J. M. Souney et al. American Geophysical Union, Fall Meeting 2018, abstract C41C-1771 .

[6] ARIANNA Collaboration, A. Anker et al. JINST 15 no. 09, (2020) P09039.

[7] L. Zhao for the ARIANNA collaboration PoS ICRC2021 (these proceedings) 1156.

[8] C. Glaser et al. European Physical Journal C80 (2020) 77.

[9] S. Barwick for the ARIANNA collaboration PoS ICRC2021 (these proceedings) 1190.

[10] S. Hallmann for the IceCube-Gen2 collaboration PoS ICRC2021 (these proceedings) 1183.

[11] C. Glaser and S. W. Barwick JINST 16 no. 05, (May, 2021) T05001.

[12] C. Glaser et al. European Physical Journal C79 no. 6, (2019) 464.

[13] S. Stjärnholm, O. Ericsson and C. Glaser PoS ICRC2021 (these proceedings) 1055. 


\section{Full Authors List: ARIANNA Collaboration}

Astrid Anker ${ }^{1}$, Pierre Baldi ${ }^{2}$, Steven W. Barwick ${ }^{1}$, Jakob Beise ${ }^{3}$, Hans Bernhoff ${ }^{4}$, Dave Z. Besson ${ }^{5,6}$, Nils Bingefors ${ }^{3}$, Maddalena Cataldo $^{7}$, Pisin Chen ${ }^{8}$, Daniel García Fernández ${ }^{9}, 7$, Geoffrey Gaswint ${ }^{1}$, Christian Glaser ${ }^{3}$, Allan Hallgren ${ }^{3}$, Steffen Hallmann ${ }^{9}$, Jordan C. Hanson ${ }^{10}$, Spencer R. Klein ${ }^{11}$, Stuart A. Kleinfelder ${ }^{12}$, Robert Lahmann ${ }^{7,1}$, Jiayi Liu ${ }^{1}$, Mitchell Magnuson ${ }^{5}$, Stephen McAleer ${ }^{2}$, Zach Meyers ${ }^{9}$, Jiwoo Nam ${ }^{8}$, Anna Nelles ${ }^{9,7}$, Alexander Novikov ${ }^{5,6}$, Manuel P. Paul ${ }^{1}$, Christopher Persichilli ${ }^{1}$, Ilse Plaisier ${ }^{9,7}$, Lilly Pyras $^{9}$, Ryan Rice-Smith ${ }^{1}$, Joulien Tatar ${ }^{13}$, Shih-Hao Wang ${ }^{8}$, Christoph Welling ${ }^{9,7}$, Leshan Zhao ${ }^{1}$

${ }^{1}$ Department of Physics and Astronomy, University of California, Irvine, CA 92697, USA.

${ }^{2}$ Department of Information and Computer Science, University of California, Irvine, CA 92697, USA.

${ }^{3}$ Uppsala University Department of Physics and Astronomy, Uppsala SE-752 37, Sweden.

${ }^{4}$ Uppsala University Department of Engineering Sciences, Division of Electricity, Uppsala SE-752 37, Sweden.

${ }^{5}$ Department of Physics and Astronomy, University of Kansas, Lawrence, KS 66045, USA.

${ }^{6}$ National Research Nuclear University MEPhI (Moscow Engineering Physics Institute), Moscow 115409, Russia.

${ }^{7}$ ECAP, Friedrich-Alexander Universität Erlangen-Nürnberg, 91058 Erlangen, Germany.

${ }^{8}$ Department of Physics and Leung Center for Cosmology and Particle Astrophysics, National Taiwan University, Taipei 10617, Taiwan. ${ }^{9}$ DESY, 15738 Zeuthen, Germany.

${ }^{10}$ Whittier College Department of Physics, Whittier, CA 90602, USA.

${ }^{11}$ Lawrence Berkeley National Laboratory, Berkeley, CA 94720, USA.

${ }^{12}$ Department of Electrical Engineering and Computer Science, University of California, Irvine, CA 92697, USA.

${ }^{13}$ Research Cyberinfrastructure Center, University of California, Irvine, CA 92697, USA.

\section{Acknowledgements}

We are grateful to the U.S. National Science Foundation-Office of Polar Programs, the U.S. National Science Foundation-Physics Division (grant NSF-1607719) for supporting the ARIANNA array at Moore's Bay, and NSF grant NRT 1633631. We acknowledge funding from the German research foundation (DFG) under grants GL 914/1-1 and NE 2031/2-1m, the Taiwan Ministry of Science and Technology, the Swedish Government strategic program Stand Up for Energy, MEPhI Academic Excellence Project (Contract No. 02.a03.21.0005) and the Megagrant 2013 program of Russia, via agreement 14.12.31.0006 from 24.06.2013. 